American Journal of Applied Sciences 9 (10): 1583-1593, 2012

ISSN 1546-9239

(C) 2012 Science Publication

\title{
Comparative Evaluation of Adaptive Filter and Neuro-Fuzzy Filter in Artifacts Removal From Electroencephalogram Signal
}

\author{
${ }^{1}$ Paulchamy Balaiah and ${ }^{2}$ Ilavennila \\ ${ }^{1}$ Department of ECE, Hindusthan Institute of Technology, Coimbatore, Tamil Nadu, India \\ ${ }^{2}$ Department Of EEE, PSG College of Technology, Coimbatore, Tamil Nadu, India
}

\begin{abstract}
Problem statement: This study presents an effective method for removing mixed artifacts (EOG-Electro-ocular gram, ECG-Electrocardiogram, EMG-Electromyogram) from the EEGElectroencephalogram records. The noise sources increases the difficulty in analyzing the EEG and obtaining clinical information. EEG signals are multidimensional, non-stationary (i.e., statistical properties are not invariant in time), time domain biological signals, which are not reproducible. It is supposed to contain information about what is going on in the ensemble of excitatory pyramidal neuron level, at millisecond temporal resolution scale. Since scalp EEG contains considerable amount of noise and artifacts and exactly where it is coming from is poorly determined, extracting information from it is extremely challenging. For this reason it is necessary to design specific filters to decrease such artifacts in EEG records. Approach: Some of the other methods that are really appealing are artifact removal through Independent Component Analysis (ICA), Wavelet Transforms, Linear filtering and Artificial Neural Networks. ICA method could be used in situations, where large numbers of noises need to be distinguished, but it is not suitable for on-line real time application like Brain Computer Interface (BCI). Wavelet transforms are suitable for real-time application, but there all success lies in the selection of the threshold function. Linear filtering is best when; the frequency of noises does not interfere or overlap with each other. In this study we proposed adaptive filtering and neuro-fuzzy filtering method to remove artifacts from EEG. Adaptive filter performs linear filtering. Neuro-fuzzy approaches are very promising for non-linear filtering of noisy image. The multipleoutput structure is based on recursive processing. It is able to adapt the filtering action to different kinds of corrupting noise. Fuzzy reasoning embedded into the network structure aims at reducing errors when fine details are processed. Results: The computational result shows that the artifacts from the EEG are removed to a great extent and this has led to the accurate analysis and diagnosis of the EEG related diseases. Conclusion: Experimental results show that the proposed neuro-fuzzy technique is very effective and performs significantly better. The fidelity of the reconstructed EEG signal is assessed quantitatively using parameters such as Signal to Noise Ratio (SNR) and Power Spectral Density (PSD). In addition we have also compared the performance of adaptive filter and neuro-fuzzy filter based on the above parameters.
\end{abstract}

Key words: ECG, adaptive filter, PSD, EOG, SNR, EEG, EMG, neuro-fuzzy filter, artifacts

\section{INTRODUCTION}

In our body, brain is one of the most important organs which is controlling every function of our body. It allows different organs in our body to communicate with each other. Such brain activity is easily measured by EEG which is a non invasive facility to find out the brain disorders. So it is possible to detect people having problems in the brain by EEG. The analysis of continuous EEG signals is complex. As a science in itself it has to be completed with its own set of perplexing nomenclature. When human beings change from one physical state to another state different waves are arising in the brain. These waves are having certain frequency which change when humans change their physical states (Paulchamy et al., 2012).Those waves are categorized as follows: Alpha waves $(7.5-14 \mathrm{~Hz})$, Beta waves (14-40 Hz), Gamma waves (above $40 \mathrm{~Hz}$ ). Theta waves (4-7.5 Hz), Delta waves (0.5-4 Hz).

The EEG can vary depending on the location of the recording electrodes. The EEG is very susceptible to various artifacts causing problems for analysis and

Corresponding Author: Paulchamy Balaiah, Department of ECE, Hindusthan Institute of Technology, Coimbatore,

Tamil Nadu, India Tel: 09500347224 
interpretation. The eye movements (EOG), the muscle activation (EMG) and heart beats (ECG) also contribute to the electrical activity recorded from the scalp and they appear as artifacts in EEG. The neurological rhthyms will get affected by the artifacts which can introduce spikes in the normal EEG output.So it is difficult to analyse EEG and complex to overcome the actual problem.

Thus it is necessary to remove artifacts from the EEG inorder to restore the actual EEG.So that it will be easy for data representation and interpretation to check whether the brain is functionioning well or not.

Croft and Barry (2006) reviews a number of methods of dealing with ocular artifacts in EEG, focusing on the relative merits of a variety of EOG correction procedures (Ahsan et al., 2010). Describes the basic concepts of wavelet analysis and other applications. We proposed an approach using adaptive filter and neuro-fuzzy filter to remove mixed artifact from EEG signals. The SNR in our approach is high. Further the ratio values are tabulated. With the help of the values we justify that the neuro-fuzzy filter removes artifacts better than adaptive filter.

\section{MATERIAL AND METHODS}

\section{Existing method:}

Principle Component Analysis: Lagerlundet.al used Principal Component Analysis (PCA) to remove the artifacts from EEG. It outperformed the regression based methods. However, PCA cannot completely separate OA from EEG, when both the waveforms have similar voltage magnitudes. PCA decomposes the leads into uncorrelated, but not necessarily independent components that are spatially orthogonal and thus it cannot deal with higher-order statistical dependencies.

Independent component analysis: An alternative approach is to use independent components analysis (ICA), which was developed in the context of blind source separation problems to obtain components that are approximately independent. ICA has been used to correct for ocular artifacts, as well as artifacts generated by other sources (Hussain et al., 2009). ICA is an extension of PCA which not only decorrelates but can also deal with higher order statistical dependencies. However, the ICA components lack the important variance maximization property possessed by the PCA components.

Canonical correlation analysis: The Canonical Correlation Analysis (CCA) is developed to overcome the disadvantages of ICA. CCA is used as a Blind Source Separation technique (BSS) for artifacts removal from EEG signal. CCA based BSS method utilizes the temporal auto correlation in the source signal as a contrast function. It measures the linear relationship between two multi-dimensional variables, by finding two bases and bases are optimal with respect to correlation. CCA method has considerable amount of spectral error and thus it cannot be implemented in real time.

Regression method: This method is based on complex regression analysis. It is suitable for handling transfer of EOG activity to EEG which can have different frequency and phase characteristics, because the regression formula is used in frequency domain. A complex regression coefficient (P (jw)) for EOG and EEG is calculated and then a common regression is removed from EEG. It is found that the complex regression coefficient $(\mathrm{P}(\mathrm{jw}))$ is used in the subtraction formula. This technique is demanding because it requires quantitative data relating to several thousand individuals. But this much data collection will take more amount of time and expensive.

Proposed method: The flowchart of the proposed method is shown in Fig. 1. In this method we include three artifacts (EOG, EMG, ECG) to the EEG signal. Now the proposed methid is to remove, these artificats using two methods which are adaptive filtering and neuro-fuzzy filter in paralell manner.

The denoised signals from both the methods are cross correlated using few parameters such as SNR and PSD. Few suggestions are made the real-time removal of Artifacts using adaptive filtering. Neuro-fuzzy approaches are very promising for non-linear filtering of noisy images. It had not been proved that they are applicable for signals. Hence in this method the noise is removed from the EEG signal by both adaptive and neuro-fuzzy filtering method and the performance of them are noted and compared. In this method, the primary input is the measured EEG and the reference input is the artifacts signal.

EEG: Brain is one of the most important organs of humans, for controlling the coordination of human muscles and nerves. The EEG is the recording of brain's electrical activity. EEG is one commonly used non-invasive facility to investigate the intricacy of human brain (Cheng, 2007). The EEG is used in the evaluation of brain disorders. It is also used to evaluate people who are having problems associated with brain. An EEG is also used to determine brain death. The analysis of continuous EEG signals is complex. As a science in itself it has to be completed with its own set of perplexing nomenclature. Different waves are categorized by the frequency of their emanations. Five types are particularly important. 
Am. J. Applied Sci., 9 (10): 1583-1593, 2012

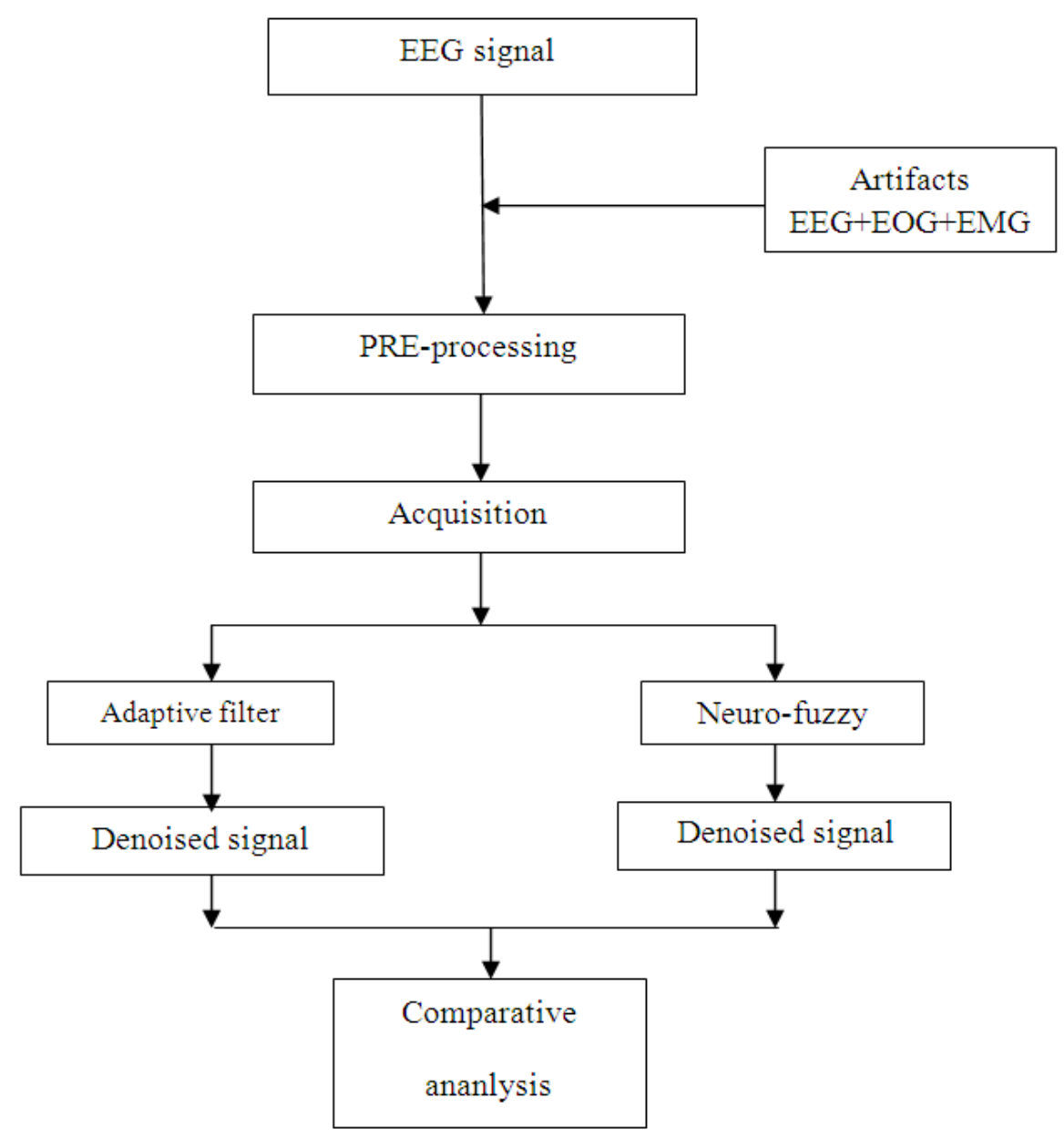

Fig. 1: Proposed method

Alpha waves $(7.5-14 \mathrm{~Hz})$, Beta waves $(14-40 \mathrm{~Hz})$, Gamma waves (above $40 \mathrm{~Hz}$ ), Theta waves $(4-7.5 \mathrm{~Hz}$ ), Delta waves $(0.5-4 \mathrm{~Hz})$.The EEG can vary depending on the location of the recording electrodes. The EEG is very susceptible to various artifacts causing problems for analysis and interpretation. The eye movements (EOG), the muscle activation (EMG) and heart beats (ECG) also contribute to the electrical activity recorded from the scalp and they appear as artifacts in EEG. The amplitude of artifacts can be quite large relative to the size of amplitude of the cortical signals of interest. The artifact removal is of prime necessity to make data interpretation and representation and to recover the signal that matches perfectly a brain functioning.

Preprocessing of EEG: The several dimensions of EEG signal may contain only empty noises. Thus Preprocessing is introduced which is nothing but the reduction of the dimensionality of EEG signal.. The purpose of preprocessing the EEG signal is to enhance the analysis on these signals. Removing artifacts and short-time high-amplitude events enable us to highlight important characteristic features in the EEG signals. The goal of signal pre-processing is to extract relevant information from the sensor responses and prepare the EEG signal for multivariate pattern analysis. There is a number of different tools and methods used for preprocessing. Some of the methods are sampling, feature extraction, transformation and normalization. Among these methods we go for feature extraction. This method pulls out specified data that is significant in some particular context and these pulled out data yields more information about the signal that can be used for further analysis and noise removal.

Adaptive filter: An adaptive filter is a computational device that attempts to model the relationship between two signals in real time in an iterative manner. An adaptive filter is defined by four aspects (Ali et al., 2010): 
- The signals are being processed by the filter

- The structure that defines show the output signal of the filter is computed from its input signal

- The parameters within this structure that can be iteratively changed to alter the filter's input-output relationship

- The adaptive algorithm that describes how the parameters are adjusted from one time instant to the next

By choosing a particular adaptive filter structure, one specifies the number and type of parameters that can be adjusted. The adaptive algorithm used to update the parameter values of the system can take on a myriad of forms and is often derived as a form of optimization procedure that minimizes an error criterion that is useful for the task at hand. In this Adaptive filter section, we present the general adaptive filtering problem and introduce the mathematical notation for representing the form and operation of the adaptive filter. We give a simple derivation of the Least Mean Square (LMS) algorithm, which is perhaps the most popular method for adjusting the coefficients of an adaptive filter and we discuss some of this algorithm's properties.

Structure of adaptive filter: The basic adaptive noise canceller scheme is the same as that illustrated in Fig. 2, where the primary signal is called "corrupted signal" and the secondary is called "reference signal" (Amble, 1987). It is assumed that the corrupted signal $d(n)$ is composed of the desired signal s (n) and noise signal $n$ $(\mathrm{n})$, which is additive and not correlated with $\mathrm{s}(\mathrm{n})$. Likewise, the references signal $x(n)$ is uncorrelated with $\mathrm{s}(\mathrm{n})$ and correlated with $\mathrm{n}(\mathrm{n})$. The reference $\mathrm{x}(\mathrm{n})$ feeds the filter to produce the output $y(n)$ that is a close estimate of $n(n)$.

NEURO-fuzzy filter: Early computational approaches could only analyze the simple systems. Many complex systems in biology, medicine were not effectively analyzed by this technique. So Soft Computing was developed. Unlike hard computing they deal with uncertainty, partial truth and approximation. This soft computing techniques include- (i) Neural Networks, (ii) Fuzzy systems and (iii) Genetic algorithm.

The Genetic algorithm is more intact with the chromosomal structures. In order to analyze the signals more effectively we go for the Neuro-Fuzzy systems. Neuro-Fuzzy system is a combination of artificial neural networks and the fuzzy logic. It is a hybrid system. Every intelligent part has its own advantage and disadvantage. For instance if we take the neural networks they are good at recognizing the patterns of signals but not good at explaining how they reach their conclusions. On the other hand, the fuzzy systems have a very good logic behind their decision but cannot automatically acquire the rules they used to make those decisions.

The above reasons are the central driving force for hybridization. The techniques are combined together to overcome the limitations of the individual technique. This cooperative approaches use neural network to make best use of certain parameters of fuzz systems and vice-versa. This neuro-fuzzy approach is very promising for non-linear although generally assumed to be the realization of a fuzzy system through connectionist network, this term is also used to describe some other configurations including:

- Deriving fuzzy rules from trained neural networks

- Fuzzy logic based tuning of neural network training parameter

- Fuzzy logic criteria for increasing a network size

- Realizing fuzzy membership function through clustering algorithm in unsupervised learning in SOMs and neural network

- Representing Fuzzification, fuzzy inference and defuzzification through multilayer feed-forward connectionist networks (Karait et al., 2009)

Structure of NEURO-fuzzy system: Two possible models of neuro-fuzzy systems are:

- In response to linguistic statements, the fuzzy interface block provides an input vector to a multilayer neural network. The neural network can be adapted (trained) to yield desired command outputs or decisions

- A multi-layered neural network drives the fuzzy inference mechanism. Fig. 3 and 4 shows the first and second model of neuro-Fuzzy Systems.

Neural networks are used to tune membership functions of fuzzy systems that are employed as decisionmaking systems for controlling equipment. Al though fuzzy logic can encode expert knowledge directly using rules with linguistic labels, it usually takes a lot of time to design and tune the membership functions which quantitatively define these linguistic labels. Neural network learning techniques can automate this process and substantially reduce development time and cost while improving performance. 


\section{Cancellation of artifacts:}

Methodology of neuro-fuzzy filter: The computational process envisioned for fuzzy neural systems is as follows. It starts with the development of a" fuzzy neuron" based on the understanding of biological neuronal morphologies, followed by learning mechanisms (Bukhari et al., 2011). This leads to the following three steps in a fuzzy neural computational process.
- Development of fuzzy neural models motivated by biological neurons

- Models of synaptic connections which incorporates fuzziness in to neural network

- Development of learning algorithms (that is the method of adjusting the synaptic weights)

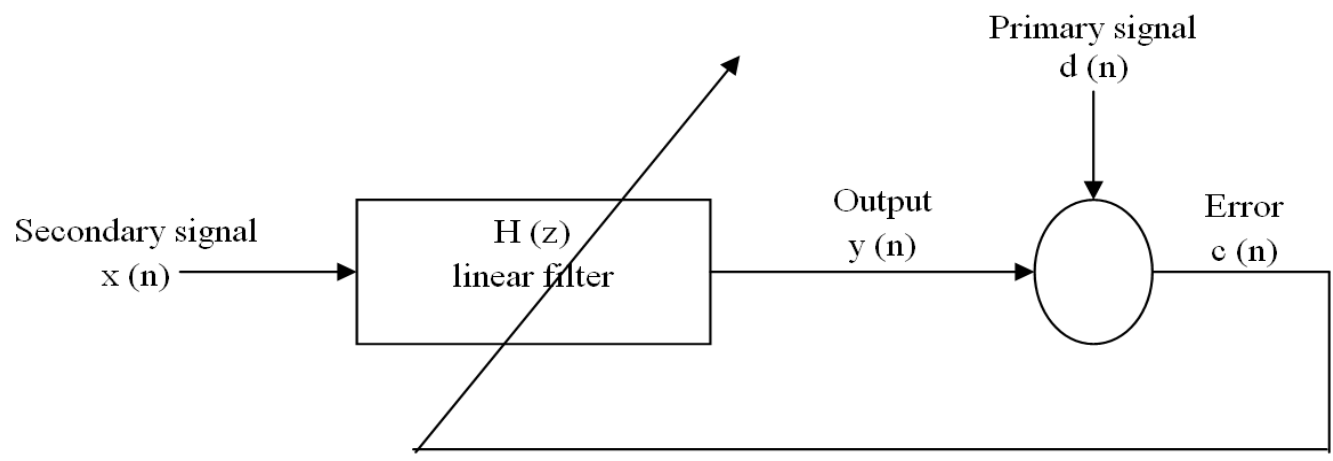

Fig. 2: Structure of Adaptive Filter

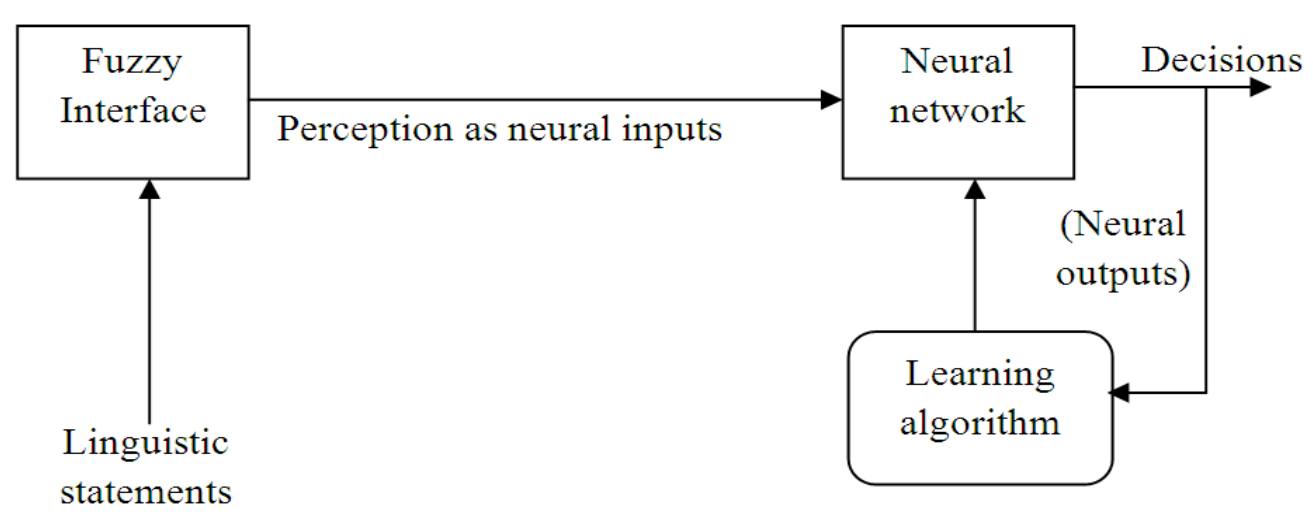

Fig. 3: First model of neuro-fuzzy systems

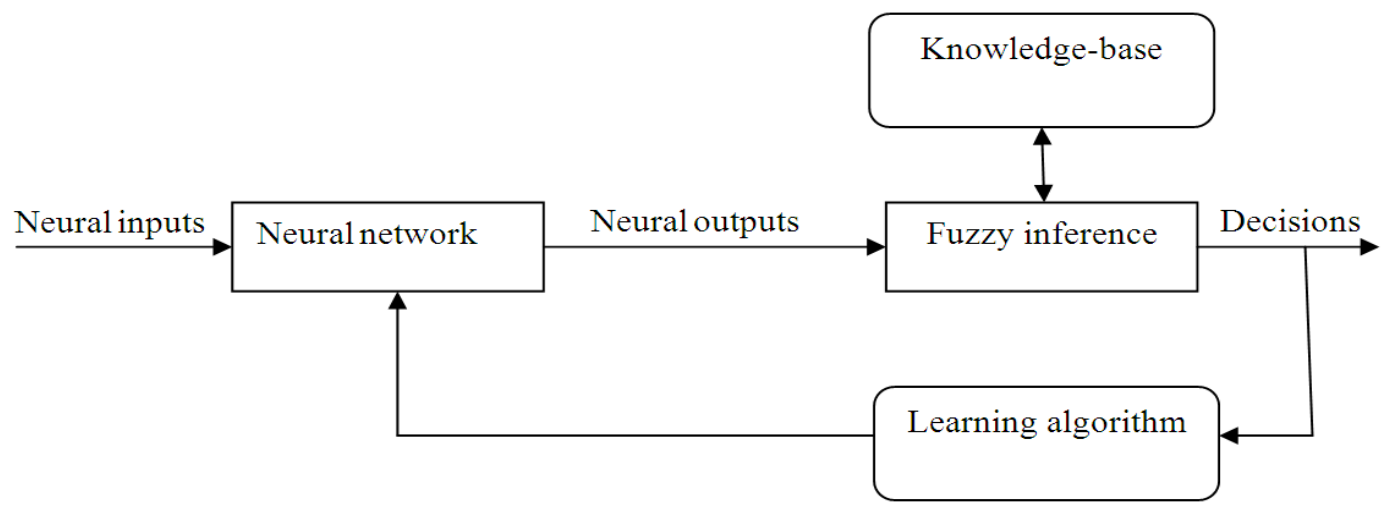

Fig. 4: Second model of neuro-fuzzy systems 


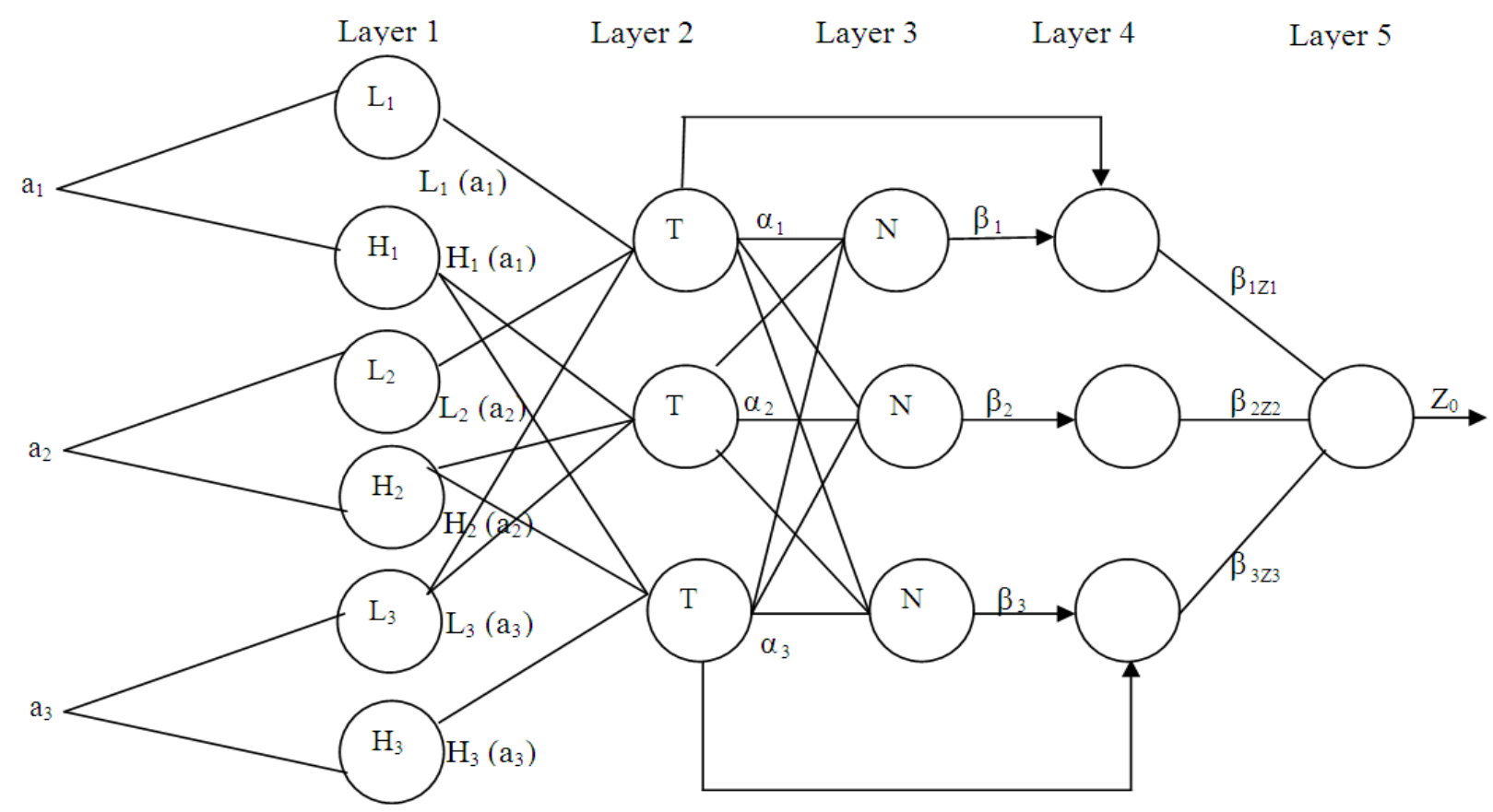

Fig. 5: Structure ofNeuro-Fuzzy Filter

The Fig. 5 gives the pictorial representation of the neuro-fuzzy filter. It includes subnet works. The circle denotes aggregation techniques. In this study, we have taken signals namely artifacts and delayed artifacts as inputs and measured EEG signal as target for training the Neuro-Fuzzy filter structure ). We have used generalized bell type as membership function for tuning the parameters. The filter has the following structure,

Layer 1: The output of the node is the degreeto which the given input satisfies the linguistic label associated to this node.

Layer 2: Each node computes the firing strength of the associated rule. The nodes of this layer are called rule nodes.

The output of top neuron is Eq. 1:

$\alpha_{1}=\mathrm{L}_{1}\left(\mathrm{a}_{1}\right) \Lambda \mathrm{L}_{2}\left(\mathrm{a}_{2}\right) \Lambda \mathrm{L}_{3}\left(\mathrm{a}_{3}\right)$

The output of the middle neuron is Eq. 2:

$\alpha_{2}=\mathrm{H}_{1}\left(\mathrm{a}_{1}\right) \Lambda \mathrm{H}_{2}\left(\mathrm{a}_{2}\right) \Lambda \mathrm{L}_{3}\left(\mathrm{a}_{3}\right)$

And the output of the bottom neuron is Eq. 3:

$\alpha_{3}=\mathrm{H}_{1}\left(\mathrm{a}_{1}\right) \Lambda \mathrm{H}_{2}\left(\mathrm{a}_{2}\right) \Lambda \mathrm{H}_{3}\left(\mathrm{a}_{3}\right)$
Layer 3: This layer is labeled as $N$ to indicate the normalization of the firing levels. The output of the top, middle and bottom neuron is the normalized firing level of the corresponding rule Eq. 4-6:

$\beta_{1}=\frac{\alpha_{1}}{\alpha_{1}-\alpha_{2}+\alpha_{3}}$

$\beta_{2}=\frac{\alpha_{3}}{\alpha_{1}-\alpha_{2}+\alpha_{3}}$

$\beta_{3}=\frac{\alpha_{3}}{\alpha_{1}-\alpha_{2}+\alpha_{3}}$

Layer 4: The output of the top, middle and bottomneuron is the product of the normalized firing Level Eq. 7-9:

$\beta_{1 \mathrm{z} 1}=\beta_{1} \mathrm{VB}^{-1}\left(\alpha_{1}\right)$

$\beta_{2 \mathrm{z} 2}=\beta_{2} \mathrm{~B}^{-1}\left(\alpha_{2}\right)$

$\beta_{3 z 3}=\beta_{3} S^{-1}\left(\alpha_{3}\right)$

Layer 5: The single node in this layer computesthe overall system output as the sum of all incoming signals Eq. 10:

$z_{0}=\beta_{1 z 1}+\beta_{3 z 2}+\beta_{3 z 3}$ 


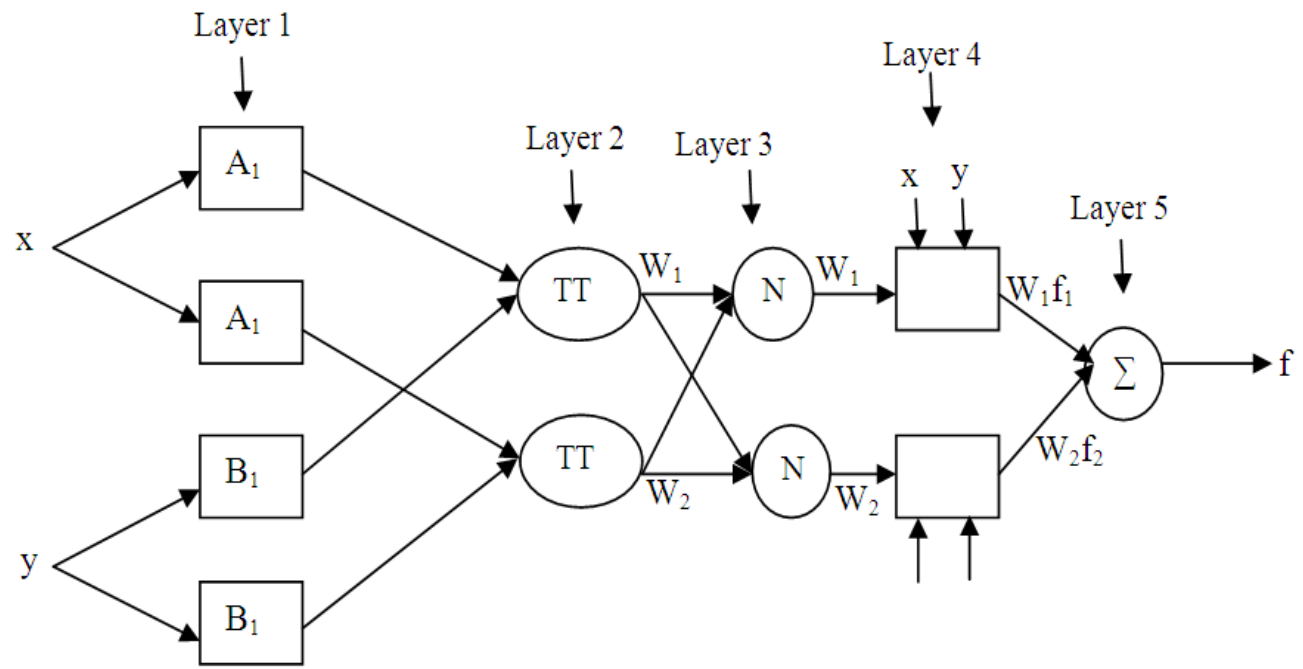

Fig. 6: Two input structure of Neuro-fuzzy filter
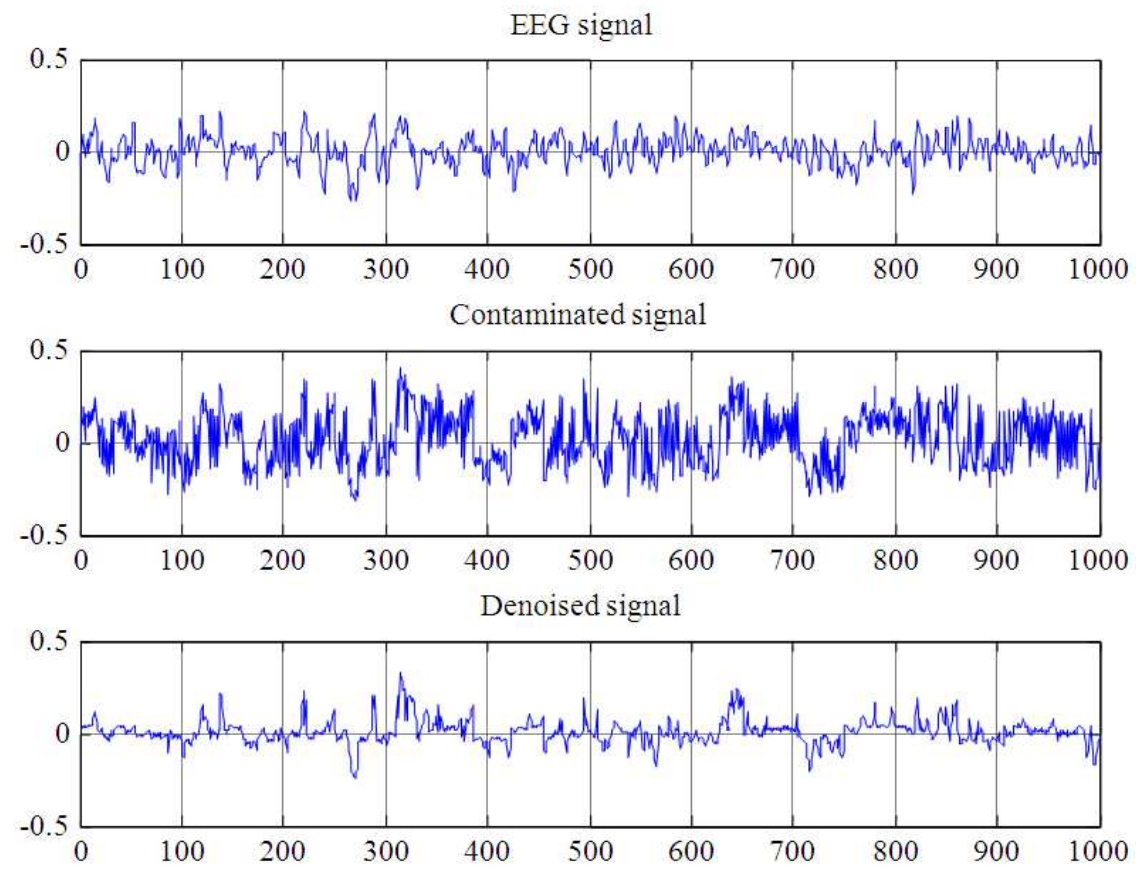

Fig. 7: Result of Adaptive filter

The general architecture of Neuro-Fuzzy filter with two inputs and one output is shown in the Fig. 6 (Bukhari et al., 2011). Assume that the Neuro-fuzzy filter has two inputs $\mathrm{x}, \mathrm{y}$ and one output $\mathrm{z}$. Figure 7 shows result of Adaptive Filter.

Rule 1: If $\mathrm{x}$ is $\mathrm{A}_{1}$ and $\mathrm{y}$ is $\mathrm{B}_{1}$, then:

$$
f_{1}=p_{1} x+q_{1} y+r_{1}
$$

Rule 2: If $x$ is $A_{2}$ and $y$ is $B_{2}$, then:

$$
\mathrm{f}_{2}=\mathrm{p}_{2} \mathrm{x}+\mathrm{q}_{2} \mathrm{y}+\mathrm{r}_{2}
$$

where, $\mathrm{p}, \mathrm{q}$ and $\mathrm{r}$ represent consequent parameters. A and $B$ are linguistic labels. Figure 8 shows the Result of Neuro-fuzzyfilter. 

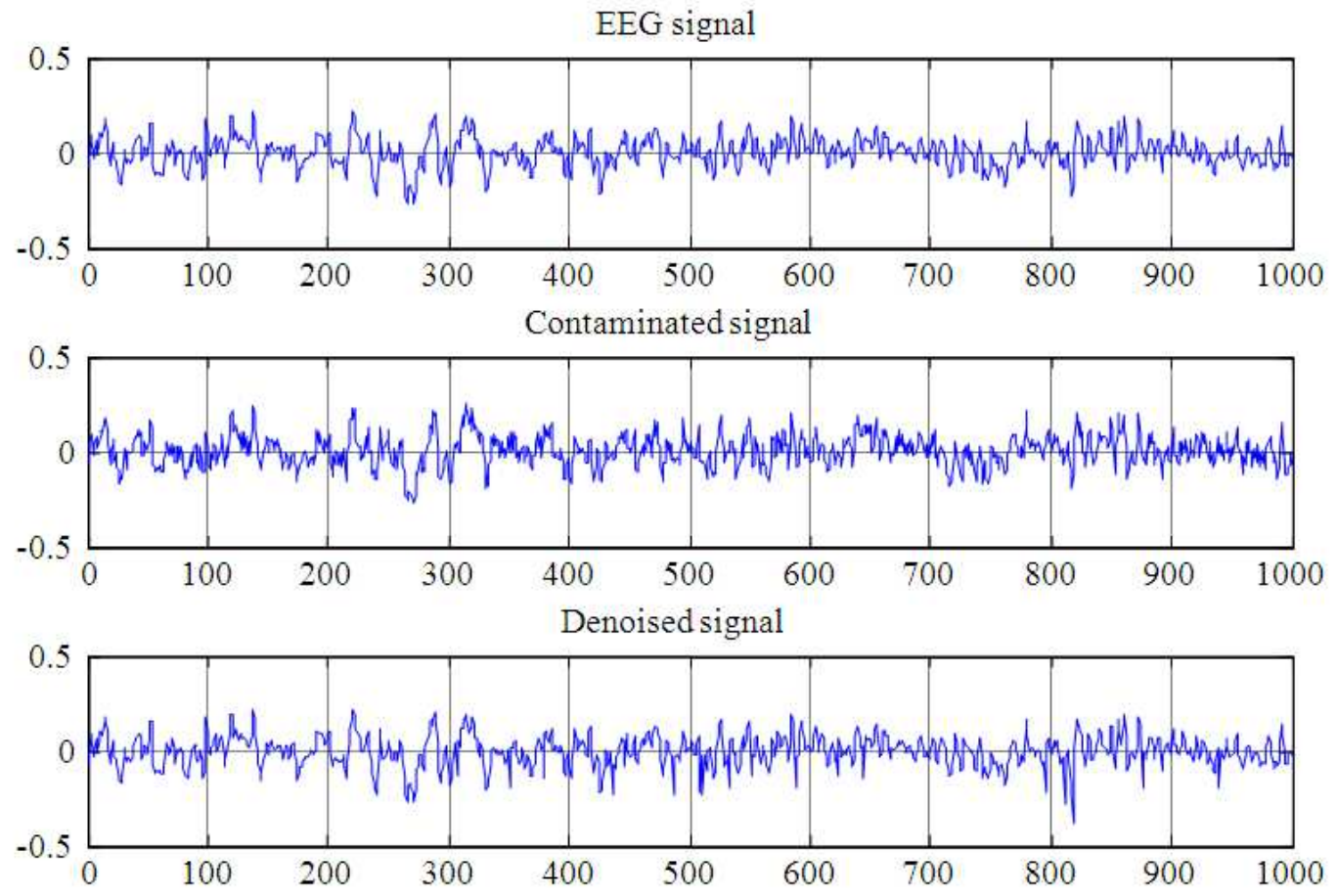

Fig. 8: Result of Neuro-fuzzy filter

Methodology of adaptive filter: The objective of an adaptive filter is to change (adapt) the coefficients of the linear filter and hence its frequency response, to generate a signal similar to the noise present in the signal to be filtered. The adaptive process involves minimization of a cost function, which is used to determine the filter coefficients. By and large, the adaptive filter adjusts its coefficients to minimize the squared error between its output and a primary signal. In stationary conditions, the filter should converge to the Wiener solution. Conversely, in non-stationary circumstances, the coefficients will change with time, according to the signal variation, thus converging to an optimum filter. In an adaptive filter, there are basically two processes:

- A filtering process, in which an output signal is the response of a digital filter. Usually, FIR filters are used in this process because they are simple and stable.

- An adaptive process, in which the transfer function $\mathrm{H} \mathrm{(z)}$ is adjusted according to an optimizing algorithm. The adaptation is directed by the error signal between the primary signal and the filter output. The most used optimizing criterion is the least mean square (LMS) algorithm.
The structure of the FIR can be represented as Eq. 11:

$y(n)=\sum_{k=0}^{1} w k(n-k)$

where, $\mathrm{L}$ is the order of the filter, $\mathrm{x}(\mathrm{n})$ is the secondary input signal, $w_{k}$ are the filter coefficients and $y(n)$ is the filter output.

The error signal e (n) is defined as the difference between the primary signal $d(n)$ and the filter output $\mathrm{y}(\mathrm{n})$, that is Eq. 12 and 13, (Furukawa, 2010):

$e(n)=d(n)-y(n)$

Where:

$y(n)=d(n)-\sum_{k=0}^{L} w_{k} x(n-k)$

The squared error is Eq. 14:

$\mathrm{e}^{2}(\mathrm{n})=\mathrm{d}^{2}(\mathrm{n})-2 \mathrm{~d}(\mathrm{n}) \sum_{\mathrm{k}=0}^{\mathrm{L}} \mathrm{w}_{\mathrm{k}} \mathrm{x}(\mathrm{n}-\mathrm{k})$

$\left[\sum \mathrm{w}_{\mathrm{k}} \mathrm{x}(\mathrm{n}-\mathrm{k})\right]^{2}$

The squared error expectation for $\mathrm{N}$ samples is given by Eq. 15 and 16: 
Am. J. Applied Sci., 9 (10): 1583-1593, 2012

$$
\begin{aligned}
& \xi(n)=E\left[e^{2}(n)\right]=\sum_{k=0}^{N} e^{2}(n) \\
& \zeta(n)=\sum_{n=1}^{N}\left[d^{2}(n)\right]-2 \sum_{k=0}^{L} w_{k} r_{d x}(n) \\
& +\sum \sum_{k=0}^{L} \mathrm{w}_{k} \mathrm{w}_{1} r_{x x}(k-1)
\end{aligned}
$$

where, $r_{d x}(n)$ and $r_{X X}(n)$ are, respectively, the crosscorrelation function between the primary and secondary input signals and the autocorrelation function of the secondary input, that is Eq. 17 and 18:

$$
\begin{aligned}
& r_{d x}(n)=\sum_{k=0}^{N} d(n) x(n-k) \\
& r_{x x}(n)=\sum_{n=0}^{N} x(n) x(n-k)
\end{aligned}
$$

The objective of the adaptation process is to minimize the squared error, which describes a performance surface. To get this goal there are different optimization techniques. In this study, we used the method of steepest descent (Teplan, 2002).

With this, it is possible to calculate the filter coefficient vector for each iteration $k$ having information about the previous coefficients and gradient, multiplied by a constant, that is Eq. 19:

$$
\mathrm{w}_{\mathrm{k}}(\mathrm{n}+1)=\mathrm{w}_{\mathrm{k}}(\mathrm{n})+\mu\left(-\nabla_{\mathrm{k}}\right)
$$

where, $\mu$ is a coefficient that the rate of adaptation.

The gradient is defined as Eq. 20:

$$
\nabla_{\mathrm{k}}=\frac{\partial\left\{\mathrm{e}^{2}(\mathrm{n})\right\}}{\partial \mathrm{W}_{\mathrm{k}}(\mathrm{n})}
$$

Substituting (20) in (19) leads to Eq. 21:

$$
\mathrm{W}_{\mathrm{k}}(\mathrm{n}+1)=\mathrm{W}_{\mathrm{k}}(\mathrm{n})-\mu \quad \frac{\partial\left\{\mathrm{e}^{2}(\mathrm{n})\right\}}{\partial \mathrm{W}_{\mathrm{k}}(\mathrm{n})}
$$

Deriving with respect to $\mathrm{w}_{\mathrm{k}}$ and replacing leads to Eq. 22 and 23:

$$
\begin{aligned}
& \mathrm{W}_{\mathrm{k}}(\mathrm{n}+1)=\mathrm{W}_{\mathrm{k}}(\mathrm{n})-2 \mu \mathrm{e}(\mathrm{n}) \quad \frac{\partial\left\{\mathrm{e}^{2}(\mathrm{n})\right\}}{\partial \mathrm{W}_{\mathrm{k}}(\mathrm{n})} \\
& \mathrm{W}_{\mathrm{k}}(\mathrm{n}+1)=\mathrm{W}_{\mathrm{k}}(\mathrm{n})-2 \mu \mathrm{e}(\mathrm{n}) \\
& \frac{\partial\left\{\mathrm{d}(\mathrm{n})-\sum_{\mathrm{k}=0}^{\mathrm{L}} \mathrm{W}_{\mathrm{k}} \mathrm{X}(\mathrm{n}-\mathrm{k})\right\}}{\partial \mathrm{W}_{\mathrm{k}}(\mathrm{n})}
\end{aligned}
$$

Since $d(n)$ and $x(n)$ are independent with respect to $\mathrm{wk}_{\mathrm{k}}$, then it shows Eq. 24:

$$
\mathrm{W}_{\mathrm{k}}(\mathrm{n}+1)=\mathrm{W}_{\mathrm{k}}(\mathrm{n})-2 \mu \mathrm{e}(\mathrm{n}) \mathrm{x}(\mathrm{n}-\mathrm{k})
$$

Equation 24 is the final description of the algorithm to compute the filter coefficients as function of the signal error $\mathrm{e}(\mathrm{n})$ and the reference input signal $\mathrm{x}(\mathrm{n})$. The coefficient $\mu$ is a constant that must be chosen for quick adaptation without losing stability. The filter is stable if $\mu$ satisfies the following condition it shows in Eq. 25:

$$
0<\mu<1 /\left(10 . \text { L.P. } P_{\mathrm{xx}}\right)
$$

where, $\mathrm{L}$ is the filter order and $\mathrm{P}_{\mathrm{XX}}$ is the power of the input signal computed as Eq. 26:

$\mathrm{P}_{\mathrm{x}} \approx \frac{1}{\mathrm{M}-1} \sum_{\mathrm{n}=0}^{\mathrm{M}-1} \mathrm{x}^{2}(\mathrm{n})$

Advantages of adaptive filters over conventional ones include preservation of components intrinsic to the EEG record.

\section{RESULTS AND DISCUSSION}

The results of EEG signal with several artifact removal using Adaptive filter and Neuro-Fuzzy filter are discussed. The following results were obtained using MATLAB software.

Case (i): Consider the real EEG signal and the mixed artifacts (EOG+EMG+ECG). Adaptive filtering is performed till the EEG signal is free from the artifacts. The figure shows (a) original EEG signal (b) noised signal (c) denoised signal.

Table 1 Summarizes the Signal to Noise ratio of Noisy signal (EEG+ECG+EMG+EOG Artifact) and Denoised Signal (Corrected EEG) using Adaptive filter. Obviously we can say that the signal to Noise Ratio of denoised signal is higher than the Noisy Signal.

Case (ii): Consider the real EEG signal and the mixed artifacts (EOG+EMG+ECG). Neuro fuzzy filtering is performed till the EEG signal is free from the artifacts. The figure shows (a) original EEG signal (b) noised signal (c) denoised signal.

Table 2 Summarizes the Signal to Noise ratio comparison of 5 trails of Noisy signal (EEG+ECG+EMG+EOG Artifact) and Denoised Signal (Corrected EEG) using Neuro-Fuzzy filter. 


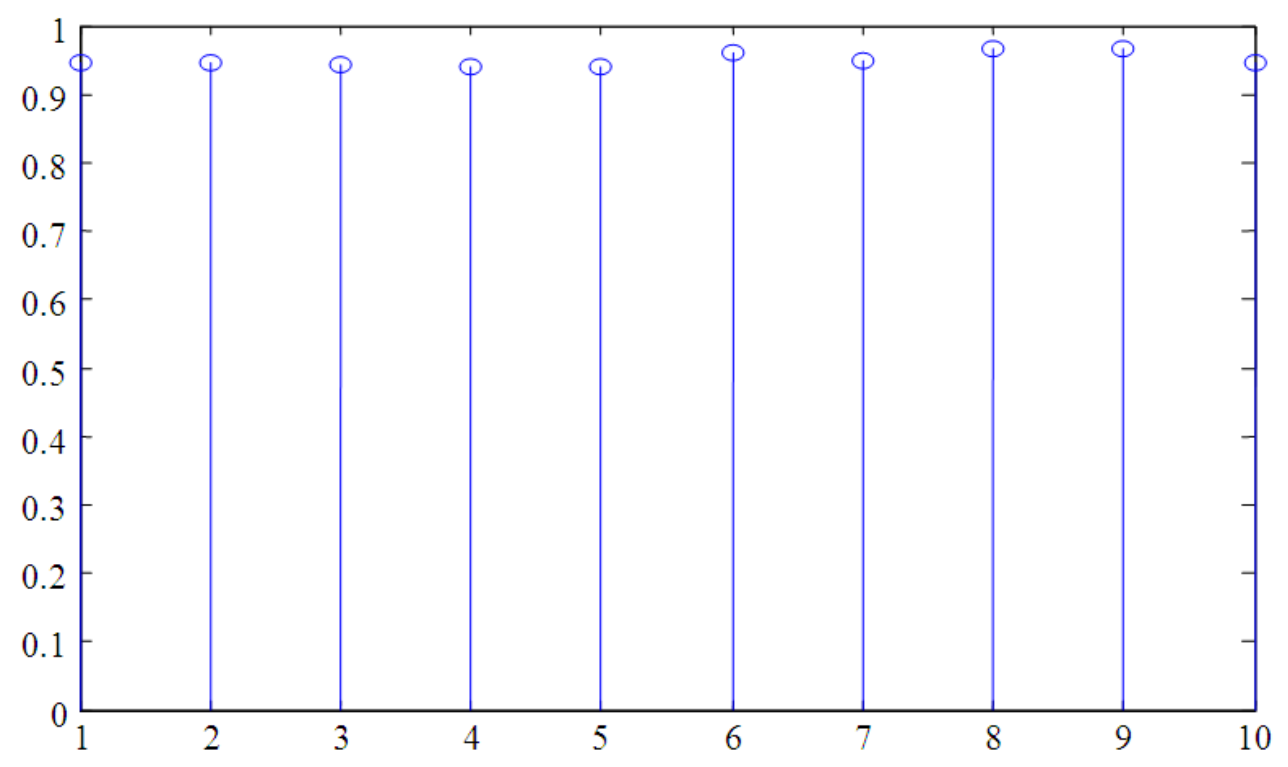

Fig. 9: Correlation Plot

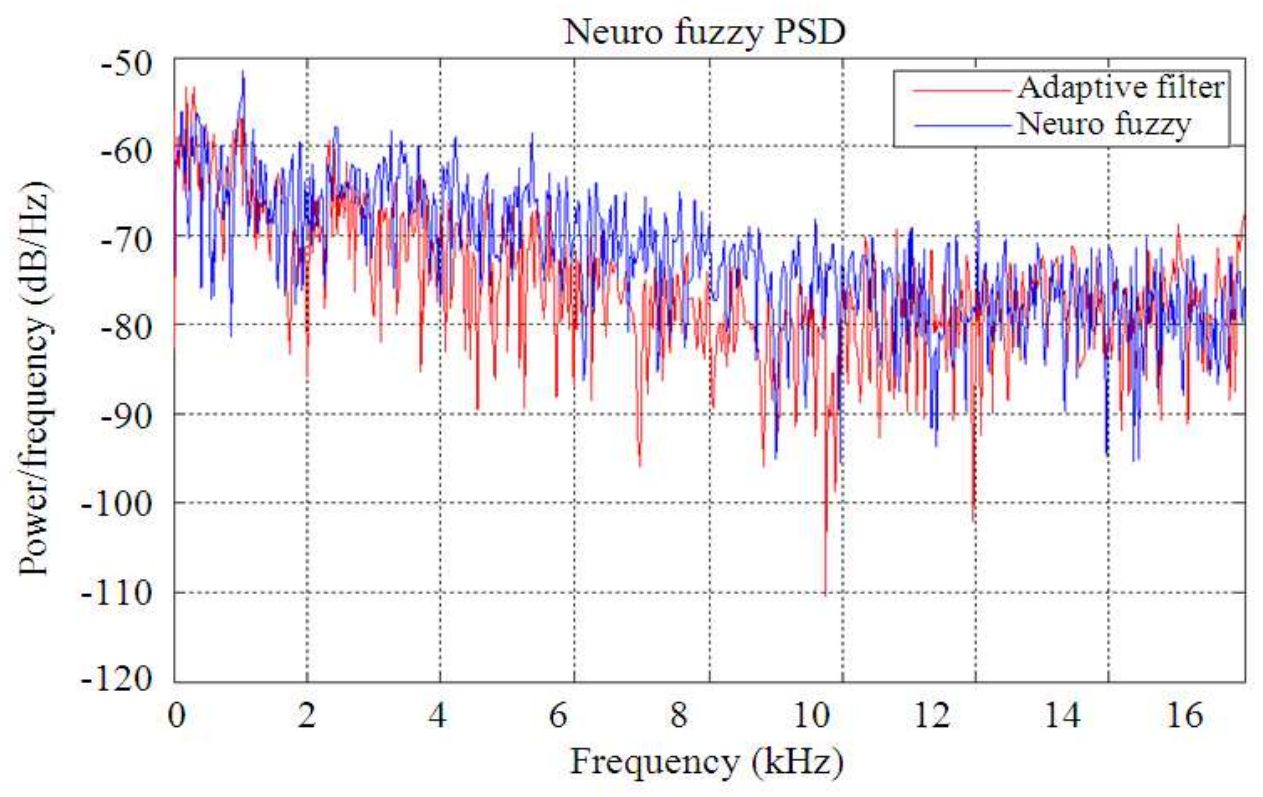

Fig. 10: Power spectral density plot

The Fig. 9 shows the correlation plot for the each trail of Noisy EEG and mixed Artifacts Removed EEG in Neuro-Fuzzy filtering. This shows how close both the signals are in terms of the Shape. (X-Axis Frequency, Y-Axis Correlation Co-efficient).

Figure 10 shows the Power Spectra of the denoised EEG in both adaptive and neuro-fuzzy filter methods (Verobyov and Cichocki, 2002). From this figure it is shown that the powers of the spectral components have been retained.

\section{CONCLUSION}

The various artifacts mixed with EEG cannot be filtered directly because they pass through the body and turn into an interference component (Schlogl et al., 2007). 
Am. J. Applied Sci., 9 (10): 1583-1593, 2012

Table 1: SNR Values for Noised and Denoised Signal

\begin{tabular}{ll}
\hline Signal & SNR value \\
\hline Noised signal & 0.51307 \\
Denoised signal & 5.00160 \\
\hline
\end{tabular}

Table 2: SNR Values for Varoius Trails of Noiised and Denoised Signal

\begin{tabular}{|c|c|c|}
\hline Trails & SNR for noised signal & SNR for denoised signal \\
\hline Trail-1 & 1.0190 & 10.3150 \\
\hline Trail-2 & 2.3579 & 9.2236 \\
\hline Trail-3 & 3.5177 & 11.3624 \\
\hline Trail-4 & 4.5408 & 11.3624 \\
\hline Trail-5 & 5.4559 & 9.2236 \\
\hline
\end{tabular}

In the proposed method EEG is subjected to noise signal and it is contaminated. Then the noise is removed by means of Adaptive filter and Neuro-fuzzy filter. The SNR ratio for both noised and denoised signal is calculated and it is observed that the SNR of the denoised signal is higher than the noised one. Also the power spectral density of the denoised signal is plotted. From the observations the performance of both adaptive filter and the Neuro-fuzzy filter is noted (Ahsan et al., 2010). By comparative study we thereby conclude that the performance of Neuro-Fuzzy filter is better than the Adaptive filter. The performance is evaluated by comparing their corresponding SNR and PSD.

\section{REFERENCES}

Ahsan, M. D.R., M.I. Ibrahimy and O.O. Khalifa, 2010. Advances in electromyogram signal classification to improve the quality of life for the disabled and aged people. J. Comput. Sci., 6: 706-715. DOI: 10.3844/jcssp.2010.706.715

Ali, A.D., P.D. Swami and J. Singhai, 2010. Modified curvelet thresholding algorithm for image denoising. J. Comput. Sci., 6: 18-23. DOI: 10.3844/jcssp.2010.18.23

Bukhari, W.M., W. Daud, R. Sudirman and C. Omar, 2011. Identification of electrooculography signals frequency energy distribution using wavelet algorithm. J. Comput. Sci., 7: 1619-1625. DOI: 10.3844/jcssp.2011.1619.1625Cheng, K., 2007. Biomedicine and informatics model of alzheimer's disease. Am. J. Biochem. Biotechnol., 3: 145-149. DOI: 10.3844/ajbbsp.2007.145.149
Croft, R.J. and R.J Barry, 2000. Removal of ocular artifacts from EEG: A review. Clin. Neurophysiol., 30: 5-9. PMID: 10740792

Furukawa, H., 2010. Adaptable user interface based on the ecological interface design concept for multiple robots operating works with uncertainty. J. Comput. Sci., 6: 904-911. DOI: 10.3844/jcssp.2010.904.911

Hussain, A., R. Ghafar, S.A. Samad and N.M. Tahir, 2009. Anomaly detection in electroencephalogram signals using unconstrained minimum average correlation energy filter. J. Comput. Sci., 5: 501506. DOI: $10.3844 /$ jcssp. 2009.501 .506

Karait, N.O.S.B., S.M. Shamsuddin and R. Sudirman, 2009. Swarm negative selection algorithm for electroencephalogram signals classification. J. Comput. Sci., 5: 995-1002. DOI: 10.3844/jcssp.2009.995.1002

Paulchamy, B., I. Vennila and J. Jaya, 2012. A qualitative analysis of independent component analysis based algorithms for the removal of artifacts from electroencephalography signals. J. Comput. Sci., 8: 287-295. DOI: 10.3844/jcssp.2012.287.295

Schlogl, A., C. Keinrath, D. Zimmermann, R. Scherer and R. Leeb et al., 2007. A fully automated correction method of EOG artifacts in EEG recordings. Clin. Neuro. Physiol., 118: 98-104. PMID: 17088100

Teplan, M., 2002. Fundamentals of EEG measurement. Measure. Sci. Rev., 2: 1-11. Verobyov, S. and A. Cichocki, 2002. Blind noise reduction for multisensory signals using ICA and subspace filtering, with application to EEG analysis. Biol. Cybernetics, 86: 293-303. DOI: 10.1007/s00422001-0298-6 